\title{
Combinatorial Regulatory Networks Provide Novel Biomarkers for Diagnosis and Therapeutics
}

\author{
Esra Gov* \\ Department of Bioengineering, Adana Science and Technology University, Turkey \\ Received: March 09, 2018; Published: March 21, 2018 \\ *Corresponding author: Esra Gov, Department of Bioengineering, Adana Science and Technology University, Adana, Turkey, \\ Email: egov@adanabtu.edu.tr
}

\section{Introduction}

One the most important discoveries of the post-genomic era is that a large fraction of the genome transcribes a heterogeneous population of non-coding RNAs (ncRNA). NcRNAs shorter than 200 nucleotides are usually identified as short/small ncRNAs - examples include PIWI-interacting RNAs, small interfering RNAs, and microRNAs (miRNAs)-whereas those longer than 200 nucleotides are classified as long ncRNAs (lncRNAs). They are part a crucial role in RNA silencing and post-transcriptional regulation of gene expression [1]. These molecules are emerging as important regulators of cellular process, such as development, differentiation, and metabolism [2]. Long and short ncRNAs not only regulate developmental processes and cell physiology, but also involved in disease states, host-pathogen interactions, and a variety of stress responses [3]. Transcriptional regulation of gene expression is often the prior process due to response of system changes, wherein the information contained in a genome is converted and then ultimately used to produce the proteins required for a given response.

It is arranged by transcriptional regulatory proteins and their target genes working in assent through a variety of mechanisms [4]. These regulators, known as transcription factors (TFs), are proteins bind to either enhancer or promoter regions of DNA adjacent to the genes that they regulate [5]. MicroRNAs (miRNAs) have arisen as another eminent class of transcription regulator component which have huge effects on protein formation at pre-transcriptional and post-transcriptional levels. Furthermore, it was found that lncRNAs get involved in transcriptional regulation; as well as [6]. And they are responsible for activation or inhibition of TFs [7]. Though determining of the disease related gene and/or ncRNA is crucial to improve knowledge about disease pathogenesis, these components remain meager in most situations to understand the complex screen underlying disease mechanisms rev [8].

Systems biology approaches may be provide integrated analysis of different experimental data sets and understanding of how alterations in regulatory networks in disease progression by using interactions of molecules (i.e: gene, protein, metabolite) at different scale (genome, RNA, proteome, metabolome level) [9-11]. On the contrary one component (i.e: TF, miRNA, IncRNA and gene) based approaches, network biology perspective is a versatile tool to evaluate interactions of all transcriptional regulatory components (i.e: mRNA and ncRNA) with comprehensive aspect. On the other hand, many studies revealed that miRNA-TF interaction play a significant role in gene expression mechanism. Arora et al. [12] Summarized that a combinatorial action of miRNAs and TFs which share similar regulatory logics and bring about a cooperative action in the gene regulatory network were determined in biological processes.

Several transcriptional regulatory network studies in which include notably miRNA-TF- target gene interaction network have been carried out. Tsang et al. [13] investigated the expression pattern between miRNAs and their target genes, and suggested that miRNAs and their targets can share common regulator(s). Gennarino et al. [14]. Were performed an analysis of human miRNA regulatory networks by focusing on the expression relationships among miRNA targets $\mathrm{Yu}$ et al. [15] Reported that the most important tumor-regulating miRNAs and TFs were highlighted from a topological point of combinatorial regulatory network of transcriptional regulation in human cancer. Recently, reconstruction of human generic transcriptional regulatory network by using experimentally verified data and demonstrated new insights on the reciprocal interplay between miRNAs, TFs and their target genes [16].

On the other hand, a lot of researchers were reported that disease or tissue specific transcriptional network such as miRNA and TF mediated regulatory networks in glioblastoma [17]. Osteosarcoma cell proliferation process [18]. Non-small celllung cancer [19] and transcriptional misregulation in reviwed in [20] Data- driven reconstruction of networks included IncRNAs have previously performed [21-23]. However, disease specific ncRNA enriched transcriptional regulatory network will be investigated 
to identify RNA biomarkers for diagnosis and treatment by using systems biology perspective. Publicly available databases of noncoding RNA such as miRBase [24], MiRDB [25] and ChIPBase [26] for miRNAs, LNCipedia.org [27] IncRNAdb [28] and NONCODE [29], for IncRNAs can be used for construction of integrative transcriptional regulatory network.

\section{Conclusion}

The crucial aim for the further studies is to develop tools and databases to identify putative functional and pathological interactions between ncRNAs, TFs and their target genes associated with various diseases. Integration of multiple data (i.e: gene expression, ncRNA and interactions data) allows us to create more holistic aspect of the disease active transcriptional regulatory networks, namely ncRNA-enriched disease networks. This can lead us to understand properties of TF-miRNA-IncRNA- their targets regulatory mechanisms and role of ncRNAs in large gene expression regulatory network. Integrative and systematic analysis of ncRNA enriched transcriptional regulation is key element to detect more reliable RNA biomarkers for diagnosis and treatment strategies.

Moreover, the discovery of novel ncRNAs, as well as their prominent role in disease specific transcriptional regulation has accentuated with respect to identify diagnostic and therapeutic targets, but to fully understand how these RNAs contribute to gene expression need to be experimental studies. Identification of the proteins and pathways regulated by these miRNA and IncRNA is likely to provide novel insights into disease pathogenesis provide novel biomarker for disease diagnosis and determine novel drug targets for therapeutic applications. However, complicated gene expression mechanisms can be solved with RNA molecules which open new exciting avenues, as our understanding of RNAs' role improves, RNA world represent us amazing knowledge.

\section{Acknowledgment}

Support by Adana Science and Technology University Scientific Research Projects Committee (BAPKO) in the context of the project 17103030.

\section{References}

1. Bartel DP (2004) MicroRNAs: genomics, biogenesis, mechanism, and function. Cell 116(2): 281-297.

2. Papait R, Kunderfranco P, Stirparo GG, Latronico MV, Condorelli G (2013) Long noncoding RNA: a new player of heart failure ? Journal of cardiovascular translational research 6(6): 876-883.

3. Amaral PP, Dinger ME, Mattick JS (2013) Non-coding RNAs in homeostasis, disease and stress responses: an evolutionary perspective. Briefings in functional genomics 12(3): 254-278.

4. Mercer TR, Mattick JS (2013) Understanding the regulatory and transcriptional complexity of the genome through structure. Genome Research 23(7): 1081-1088.

5. Yu H, Gerstein M (2006) Genomic analysis of the hierarchical structure of regulatory networks Proc Natl Acad Sci 103(40): 14724-14731.

6. Mariner PD, Walters RD, Espinoza CA, Drullinger LF, Wagner SD, et al. (2008) Human Alu RNA is a modular transacting repressor of mRNA transcription during heat shock. Molecular Cell 29(4): 499-509.

7. Willingham AT, Orth AP, Batalov S, Peters EC, Wen BG, et al. (2005) A strategy for probing the function of noncoding RNAs finds a repressor of NFAT. Science 309(5740): 1570-1573.

8. Gov E, Kori M, Arga KY (2017) RNA based ovarian cancer research from 'a gene to systems biomedicine' perspective. Systems Biology in Reproductive Medicine 63(4): 219-238.

9. Kori M, Gov E, Arga KY (2016) Molecular Signatures of Ovarian Diseases: Insights from Network Medicine Perspective. Systems Biology in Reproductive Medicine 62(4): 266-282.

10. Gov E, Arga KY (2017) Differential co-expression analysis reveals a novel prognostic gene module in ovarian cancer. Scientific Reports p. 7.

11. Gov E, Kori M, Arga KY (2017) Multiomics Analysis of Tumor Microenvironment Reveals Gata2 and miRNA-124-3p as Potential Novel Biomarkers in Ovarian Cancer. OMICS: A Journal of Integrative Biology 21(10): 603-615.

12. Arora S, Rana R, Chhabra A, Jaiswal A, Rani V (2013) miRNAtranscription factor interactions: a combinatorial regulation of gene expression. Molecular Genetic Genomics 288(3-4): 77-87.

13. Tsang J, Zhu J, van Oudenaarden A (2007) MicroRNA-mediated feedback and feedforward loops are recurrent network motifs in mammals. Molecular Cell 26(5): 753-767.

14. Gennarino VA, D Angelo G, Dharmalingam G, Fernandez S, Russolillo $G$, et al. (2012) Identification of microRNA-regulated gene networks by expression analysis of target genes. Genome Research 22(6): 11631172.

15. Yu H, Tu K, Wang YJ, Mao JZ, Xie L, et al. (2012) Combinatorial network of transcriptional regulation and microRNA regulation in human cancer. BMC Systems Biology 12(6): 61.

16. Gov E, Arga KY (2016) Interactive cooperation and hierarchical operation of microRNA and transcription factor crosstalk in human transcriptional regulatory network. IET Systems Biology 10(6): 219-228.

17. Sun J, Gong X, Purow B, Zhao Z (2012) Uncovering MicroRNA and Transcription Factor Mediated Regulatory Networks in Glioblastoma. PLoS Computational Biology 8(7): 1002488.

18. Poos K, Smida J, Nathrath M, Maugg D, Baumhoer D, et al. (2013) How microRNA and transcription factor co-regulatory networks affect osteosarcoma cell proliferation. PLoS Computational Biology 9(8): 1003210.

19. Mitra R, Edmonds MD, Sun J, Zhao M, Yu H, et al. (2014) Reproducible combinatorial regulatory networks elucidate novel oncogenic microRNAs in non-small cell lung cancer. RNA 20(9):1356-1368.

20. Lee TI, Young RA (2013) Transcriptional regulation and its misregulation in disease. Cell 152(6): 1237-1251.

21. Li JH, Liu S, Zhou H, Qu LH, Yang JH (2014) starBase v2.0: decoding miRNAceRNA, miRNA-ncRNA and protein-RNA interaction networks from large-scale CLIP-Seq data. Nucleic Acids Research 42: 92-97.

22. Wright PR, Georg J, Mann M, Sorescu DA, Richter AS, et al. (2014) Copra RNA and IntaRNA: predicting small RNA targets, networks and interaction domains. Nucleic Acids Research 42: 119-23.

23. Bonnici V, Russo F, Bombieri N, Pulvirenti A, Giugno R (2014) Comprehensive reconstruction and visualization of non-coding regulatory networks in human. Frontiers Bioengineering Biotechnology 2: 69 .

24. Kozomara A, Griffiths Jones S (2013) miRBase: annotating high confidence microRNAs using deep sequencing data. Nucleic acids Research 42: 68-73.

25. Wang X (2008) miRDB: a microRNA target prediction and functional annotation database with a wiki interface. RNA 14(6): 1012-1017.

26. Yang JH, Li JH, Jiang S, Zhou H, Qu LH (2013) ChIPBase: a database for decoding the transcriptional regulation of long non-coding RNA and microRNA genes from ChIP-Seq data. Nucleic acids Research 41. 
27. Volders PJ, Verheggen K, Menschaert G, Vandepoele K, Martens L, et al. (2015) An update on LNCipedia: a database for annotated human IncRNA sequences. Nucleic acids Research 43: 174-D180.

28. Quek XC, Thomson DW, Maag JL, Bartonicek N, Signal B, et al. (2014) lncRNAdb v2. 0: expanding the reference database for functional long noncoding RNAs. Nucleic acids Research 43: 168 -178.

\section{(C) (i) This work is licensed under Creative}

Submission Link: https://biomedres.us/submit-manuscript.php
29. Zhao Y, Li H, Fang S, Kang Y, Wu W, et al. (2016) NONCODE 2016: an informative and valuable data source of long non-coding RNAs. Nucleic Acids Research 44: 203-208.

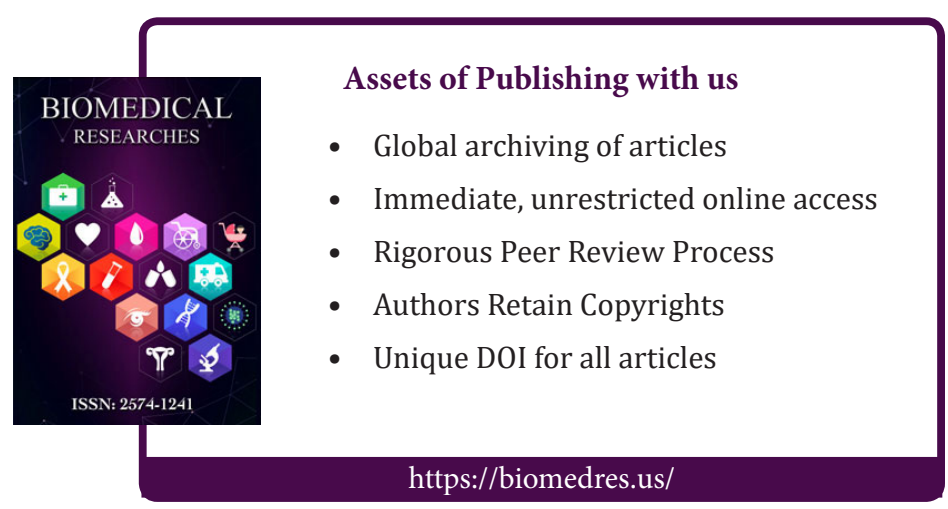

